\title{
Alkali/Surfactant/Polymer Flooding in the Daqing Oilfield Class II Reservoirs Using Associating Polymer
}

\author{
Ru-Sen Feng, ${ }^{1,2}$ Yong-Jun Guo, ${ }^{1,2}$ Xin-Min Zhang, ${ }^{1,2}$ Jun Hu, ${ }^{1,2}$ and Hua-Bing Li ${ }^{1,2}$ \\ ${ }^{1}$ State Key Laboratory of Oil and Gas Reservoirs Geology and Exploration, Southwest Petroleum University, \\ Chengdu, Sichuan 610500, China \\ ${ }^{2}$ School of Chemistry and Chemical Engineering of Southwest Petroleum University, Chengdu, Sichuan 610500, China \\ Correspondence should be addressed to Ru-Sen Feng; gs_frs@163.com and Yong-Jun Guo; gyfzgyj@126.com
}

Received 4 April 2013; Accepted 20 June 2013

Academic Editor: Ibnelwaleed Ali Hussien

Copyright (C) $2013 \mathrm{Ru}$-Sen Feng et al. This is an open access article distributed under the Creative Commons Attribution License, which permits unrestricted use, distribution, and reproduction in any medium, provided the original work is properly cited.

\begin{abstract}
Hydrophobically modified associating polyacrylamide (HAPAM) has good compatibility with the Daqing heavy alkylbenzene sulfonate surfactant. The HAPAM alkali/surfactant/polymer (ASP) system can generate ultralow interfacial tension in a wide range of alkali/surfactant concentrations and maintain stable viscosity and interfacial tension for 120 days. The HAPAM ASP system has good injectivity for the Daqing class II reservoirs $\left(100-300 \times 10^{-3} \mu \mathrm{m}^{2}\right)$ and can improve oil recovery by more than $25 \%$ on top of water flooding. In the presence of both the alkali and the surfactant, the surfactant interacts with the associating groups of the polymer to form more micelles, which can significantly enhance the viscosity of the ASP system. Compared with using HPAM $(\mathrm{Mw}=2.5 \mathrm{MDa})$, using HAPAM can reduce the polymer use by more than $40 \%$.
\end{abstract}

\section{Introduction}

ASP flooding is the chemical flooding method that gives the highest oil recovery rate [1-5]. Despite the controversies on its technical and economic feasibilities [6, 7], pilot on-site experiments and examination of its industrial application are continuously carried out [8-12]. The technical issues of ASP flooding mainly include (1) formation damage due to the use of strong base, (2) scale buildup in the injection and production equipment, and (3) difficulties in processing the produced fluid [13-15]. In addition, compared with the already industrialized polymer flooding technology, ASP flooding is considerably more costly [10], mainly because the used polymer has poor resistance against alkali. Currently, new trends in ASP flooding include (1) the use of alkali-resistant polymer to reduce costs [16]; (2) developing weak-alkali ASP flooding system to alleviate problems associated with using strong base [17]; (3) developing ASP flooding system suitable for reservoirs with low/medium permeability. Field test indicated that hydrophobically modified associating polyacrylamide (HAPAM) can be a suitable flooding agent for high-temperature high-salinity reservoirs due to its excellent characteristics in temperature, salt, and shear resistance and so forth, [18]. Nevertheless, the ASP flooding system based on HAPAM has not been reported yet. This paper describes the latest research progress on using strongly basic ASP flooding system consisting of the HAPAM associating polymer at the Daqing Class II reservoirs $\left(100-300 \times 10^{-3} \mu \mathrm{m}^{2}\right)$.

\section{Experimental}

2.1. Materials. HAPAM (Figure 1) was synthesized according to the literature [19] using $0.5 \% \mathrm{~mol} / \mathrm{mol}$ cetyl dimethylallyl ammonium chloride $\left(\mathrm{C}_{16}\right.$ DMAAC) as the hydrophobic monomer. The synthesized HAPAM has an intrinsic viscosity of $1388 \mathrm{~mL} / \mathrm{g}$ and a hydrolysis degree of $24.5 \%(\mathrm{w} / \mathrm{w})$.

HPAM (average molecular mass, $25,000 \mathrm{kDa}$; hydrolysis degree, $21.6 \% \mathrm{w} / \mathrm{w})$ was provided by Daqing Refining and Chemical Company. $\mathrm{C}_{16}$ DMAAC was supplied by Southwest Petroleum University. Other reagents were purchased from Chengdu Kelong Chemical Reagents Corporation (China). All reagents were used as received without further purification. Heavy alkylbenzene sulfonate and the crude oil ( $\rho=$ $0.85 \mathrm{~g} / \mathrm{cm}^{3}$ ) were obtained from the Daqing oilfield.

To simulate the injected water in the pilot test, inorganic salts were added to distilled water and the solution was used 


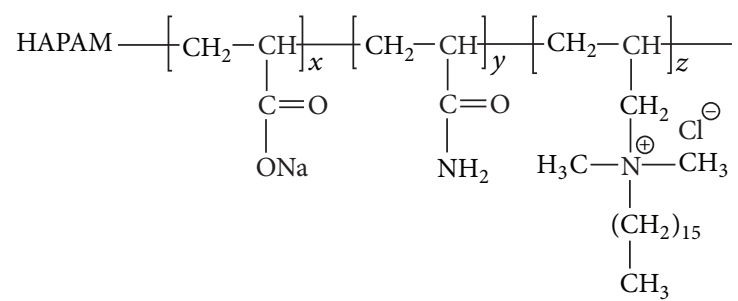

FIgURE 1: The structure of HAPAM.

TABLE 1: Composition of the simulated injection water.

\begin{tabular}{lc}
\hline Ion style & Ion concentration $\left(\mathrm{mg} \mathrm{L}^{-1}\right)$ \\
\hline $\mathrm{K}^{+}$and $\mathrm{Na}^{+}$ & 1044.87 \\
$\mathrm{Ca}^{2+}$ & 56.51 \\
$\mathrm{Mg}^{2+}$ & 24.90 \\
$\mathrm{HCO}_{3}{ }^{-}$ & 2351.91 \\
$\mathrm{SO}_{4}{ }^{2-}$ & 148.12 \\
$\mathrm{Cl}^{-}$ & 256.46 \\
\hline Total dissolved substance (TDS) & 3882.77 \\
\hline
\end{tabular}

in the subsequent experiments. The composition and salinity of the simulated injection water are given in Table 1. No precipitate was present in any formation water.

2.2. Solution Preparation. The stock solution of the surfactant or polymer $(5000 \mathrm{mg} / \mathrm{L})$ was prepared by dissolving the surfactant or polymer in brine. The ASP dilute solution was prepared by mixing the stock solutions to obtain the desired surfactant and polymer concentrations.

2.3. Measurement of Oil/Water Interfacial Tension. The oil/water interfacial tension between the solution and crude oil was measured using a Texas-500C spinning drop tensiometer (Bowing, USA) for $30 \mathrm{~min}$ at $45^{\circ} \mathrm{C}$. The instrument could automatically record the interfacial tension with an image monitoring device and an image acquisition software.

2.4. Measurement of Viscosity. Solution viscosity was measured using a Brookfield DV-III viscometer (Brookfield, USA) at $45^{\circ} \mathrm{C}$ with a shear rate of $7.34 \mathrm{~s}^{-1}$.

2.5. Injectivity Test. The cores saturated with brine were inserted into three core holders connected in series, each having a test point. The flooding solutions were injected into the cores at a constant rate of $1.0 \mathrm{~mL} / \mathrm{min}$ until the pressure stabilized. Subsequently, brine was injected into the cores that had absorbed the polymer and the surfactant, and the experiment was finished when the pressure drop stabilized across the cores. All tests were run at $45^{\circ} \mathrm{C}$. The pressures were recorded by a data terminal.

2.6. Core Flooding Test. Crude oil was injected continuously with a positive-displacement pump and an air bath held at $45^{\circ} \mathrm{C}$ until no more water was produced. Water flooding was then started until initial oil saturation was reached. Afterwards, chemical flooding was carried out by injecting a $0.3 \mathrm{PV}$ displacement plug and then flooding with chase water

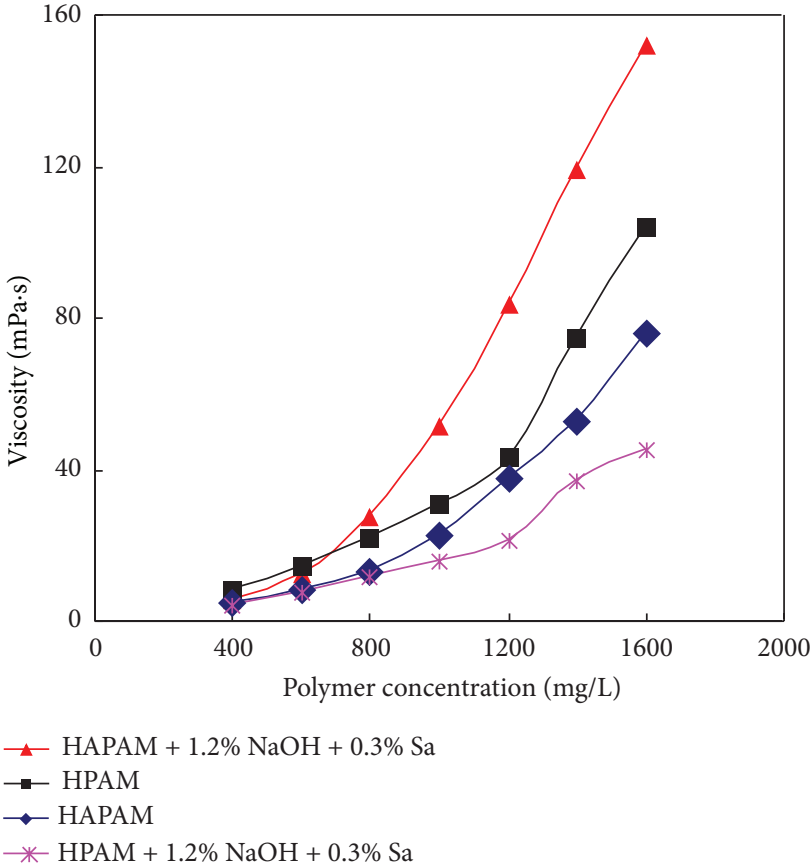

FIGURE 2: Viscosity-concentration curves of four flooding systems.

until the water cut of the produced fluid approached $98 \%$. The oil recoveries and water cuts were calculated every $0.15 \mathrm{PV}$ during the experiment.

\section{Results and Discussion}

3.1. Viscosity Enhancement. Figure 2 shows that, in the presence of alkali and surfactant, the HAPAM ASP flooding system has significantly better viscosity than using HPAM $(\mathrm{Mw}=2.5 \mathrm{MDa})$ and can reduce the polymer use by more than $40 \%$.

3.2. Compatibility between the Associating Polymer and the Surfactant. Figures 3 and 4 show the interfacial tension of the ASP systems containing HAPAM and HPAM, respectively.

The experimental comparison of the dynamic interfacial tension of the two ASP flooding systems is shown in Figure 5.

The results in Figures 3 and 4 indicate that the associating polymer HAPAM has better compatibility with heavy alkylbenzene sulfonate than HPAM and can enable ultralow interfacial tension in a wide alkali/surfactant concentration range. Figure 5 shows that HAPAM can quickly generate ultralow interfacial tension with heavy alkylbenzene sulfonate and remain stable for $120 \mathrm{~min}$.

3.3. Aging Stability of HAPAM ASP Flooding System. Figure 6 shows that, within an aging period of 120 days, the viscosity of the HAPAM ASP system was always greater than $40 \mathrm{mPa} \cdot \mathrm{s}$ and the interfacial tension remained stable.

3.4. Conductivity in Porous Media. The injectivity and conductivity of the sample were evaluated by the core flow experiment using three cores connected in series. The water 


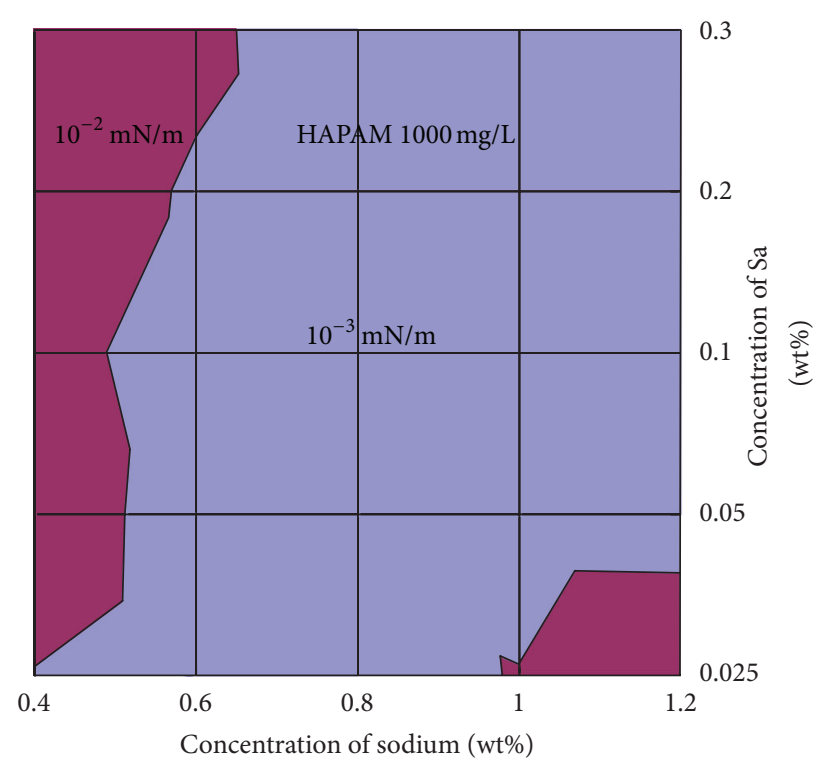

FIGURE 3: Interfacial tension of the ASP flooding system containing HAPAM.

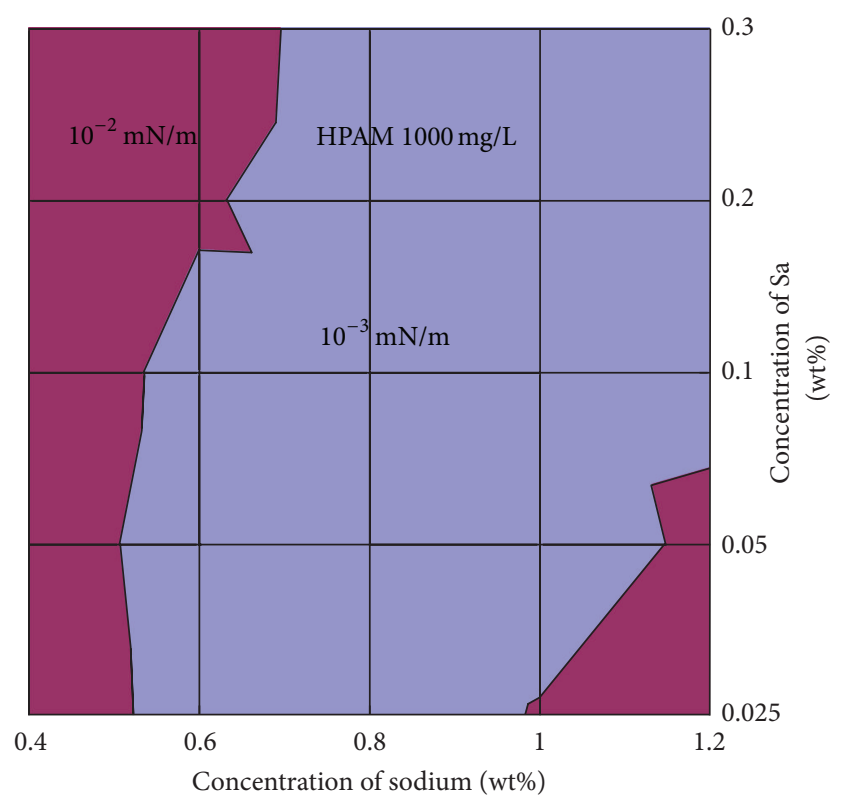

FIGURE 4: Interfacial tension of the ASP flooding system containing HPAM.

permeability of the three cores was $249 \times 10^{-3} \mu \mathrm{m}^{2}, 270 \times$ $10^{-3} \mu \mathrm{m}^{2}$, and $256 \times 10^{-3} \mu \mathrm{m}^{2}$, respectively, the concentration of the associating polymer was $1000 \mathrm{mg} / \mathrm{L}$, the surfactant concentration was $0.3 \%$, and the $\mathrm{NaOH}$ concentration was $1.2 \%$.

Figure 7 shows that the HAPAM ASP system can penetrate into the $200 \times 10^{-3} \mu \mathrm{m}^{2}$ core. The pressure gradient between the measurement points is relatively uniform, which suggests good pressure-conduction performance.

3.5. Core Flooding Experiment. Core flooding experiment was carried out using the HAPAM ASP system $(1000 \mathrm{mg} / \mathrm{L}$

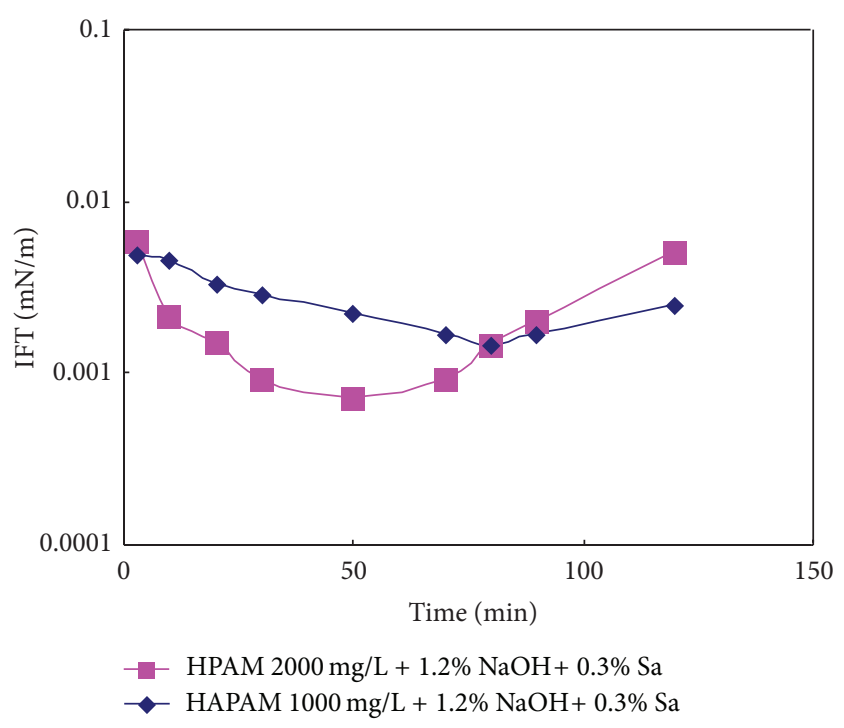

Figure 5: Dynamic interfacial tension of the two ASP systems.

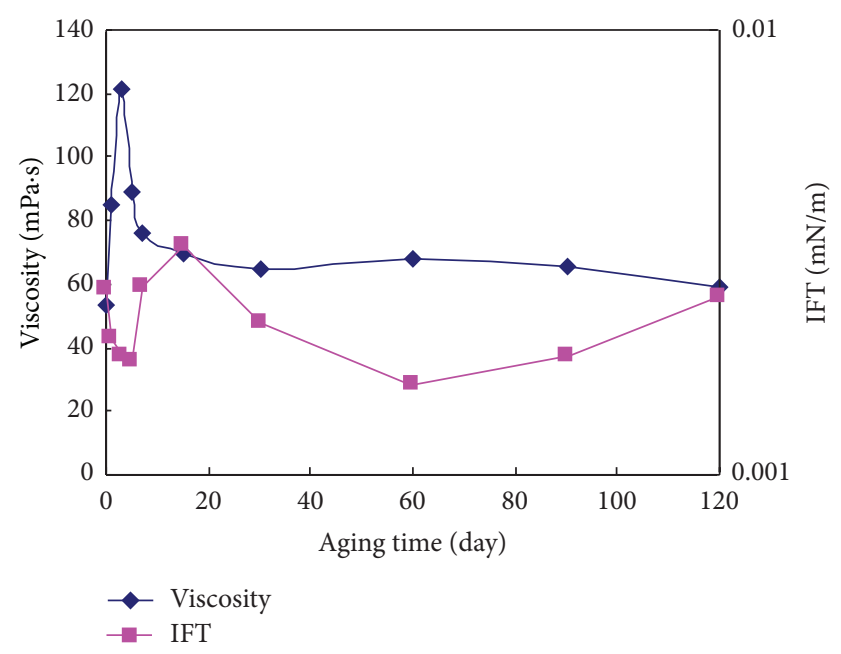

FIGURE 6: Variation of viscosity and interfacial tension with time.

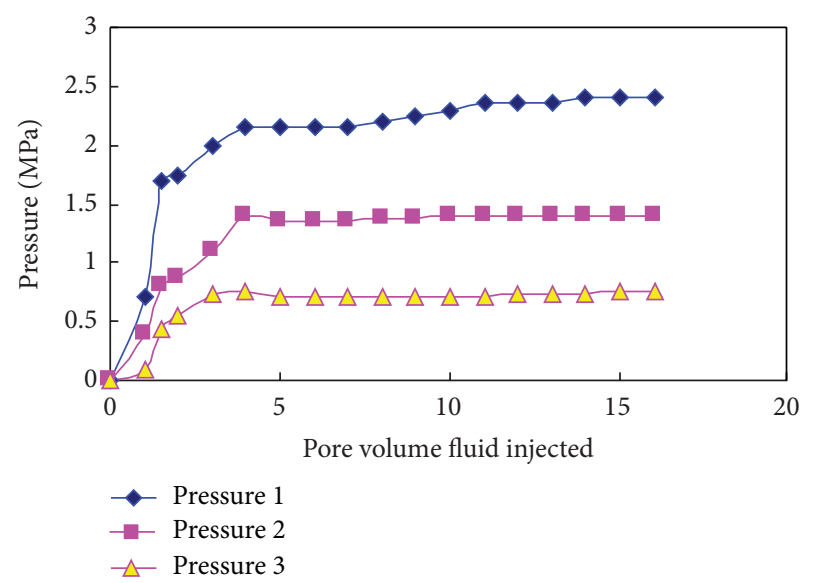

FIGURE 7: Conductivity of the HAPAM ASP flooding system in porous medium. 


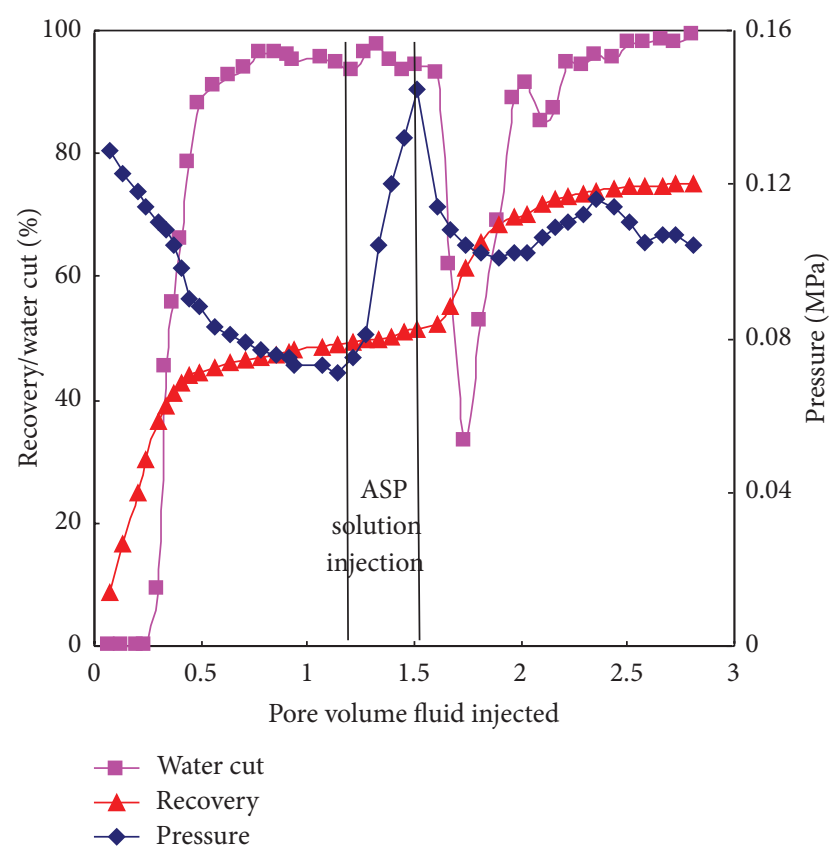

FIGURE 8: Flooding curve of the ASP flooding system.

associating polymer HAPAM $+1.2 \% \mathrm{NaOH}+0.3 \%$ heavy alkylbenzene sulfonate) and $30 \mathrm{~cm}$ homogeneous square cores $\left(184 \times 0^{-3} \mu \mathrm{m}^{2}\right)$. The experimental results are shown in Figure 8.

The results in Figure 8 show that using $0.3 \mathrm{PV}$ ASP flooding fluid can further improve oil recovery by $26 \%$ on top of water flooding.

3.6. Performance of ASP System under Field Conditions. The on-site injection water and surfactant were used to further investigate the performance of the HAPAM ASP flooding system.

The mother liquor was prepared at $20^{\circ} \mathrm{C}$ for a dissolution time of $2 \mathrm{~h}$ using on-site water of the oil production plant and the on-site dehydrated crude oil. The surfactant (effective content $50 \%$ ) and $\mathrm{NaOH}$ were all on-site industrial products.

3.6.1. Viscosity. The ASP systems containing HAPAM and $\operatorname{HPAM}(\mathrm{Mw}=2.5 \mathrm{MDa})$ were prepared. The polymer concentration was $1000 \mathrm{mg} / \mathrm{L}$, the surfactant concentration was $0.3 \%$, and the $\mathrm{NaOH}$ concentration was $1.2 \%$. The change of the different systems with the concentration is shown in Figure 9.

Figure 9 shows that, after the addition of base and surfactant, the HAPAM ASP system has clearly better viscosity under field conditions compared with other systems.

3.6.2. Interfacial Tension. The interfacial tension was measured at a fixed HAPAM concentration of $1000 \mathrm{mg} / \mathrm{L}$ and varying concentrations of surfactant and base. The experimental results are shown in Figure 10.

3.7. Discussions on the Viscosity Enhancement of the HAPAM ASP System. The prominent feature of the HAPAM ASP

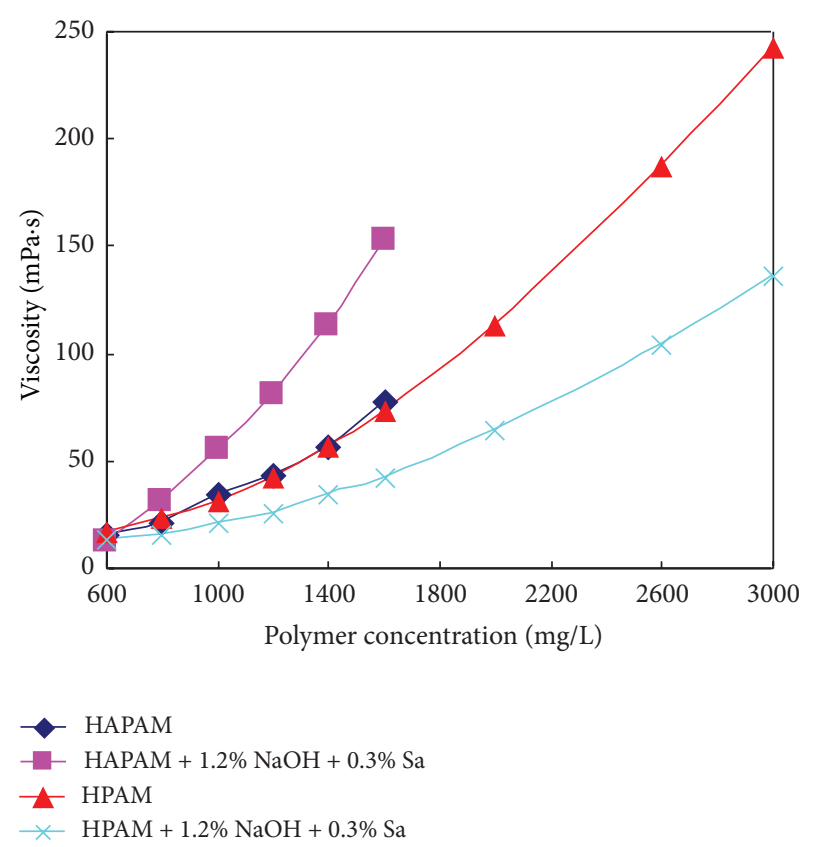

FIGURE 9: Viscosity-concentration curves of four flooding systems (in on-site injection water).

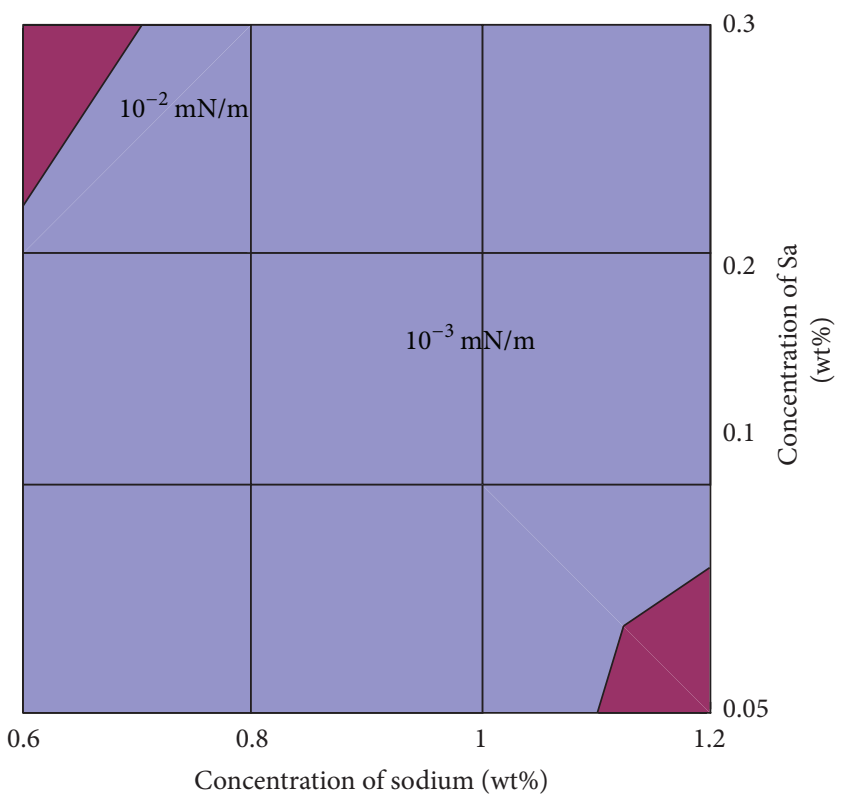

FIGURE 10: Interfacial tension of the HAPAM ASP system (in on-site injection water).

system is that, in the presence of both the base and the surfactant, the viscosity of the ASP system is significantly higher than that of the polymer solution alone, indicating a viscosity enhancing effect of the associating polymer under the given conditions. In this paper, the mechanism of this viscosity enhancement is analyzed and discussed (see Figure $11)$. 


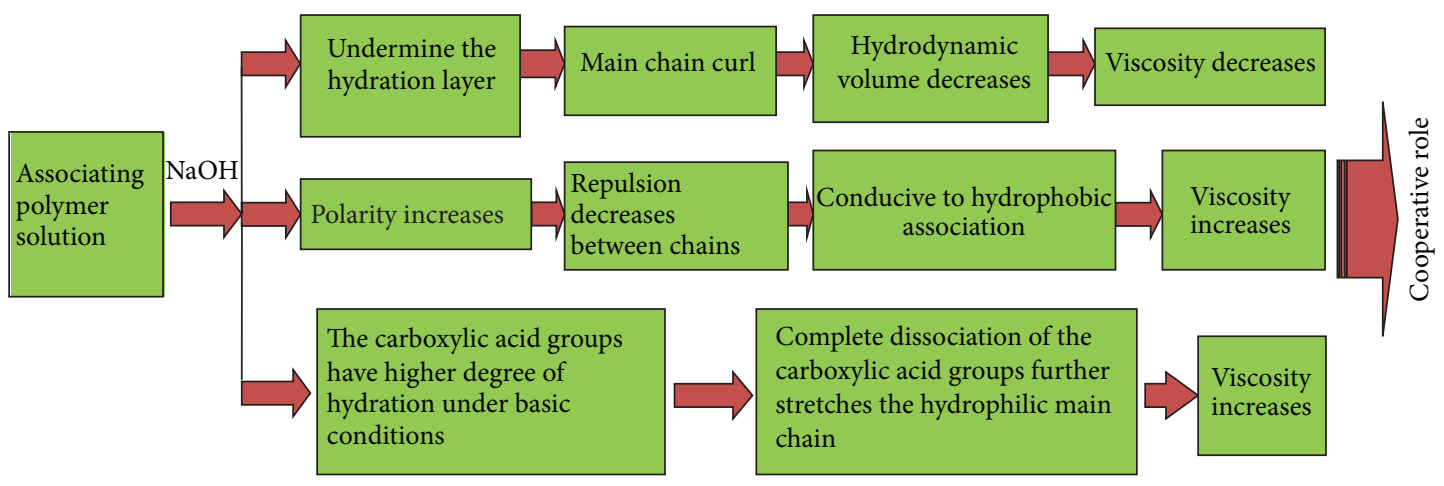

FIGURE 11: The effect of alkali on the viscosity of the associating polymer solution.

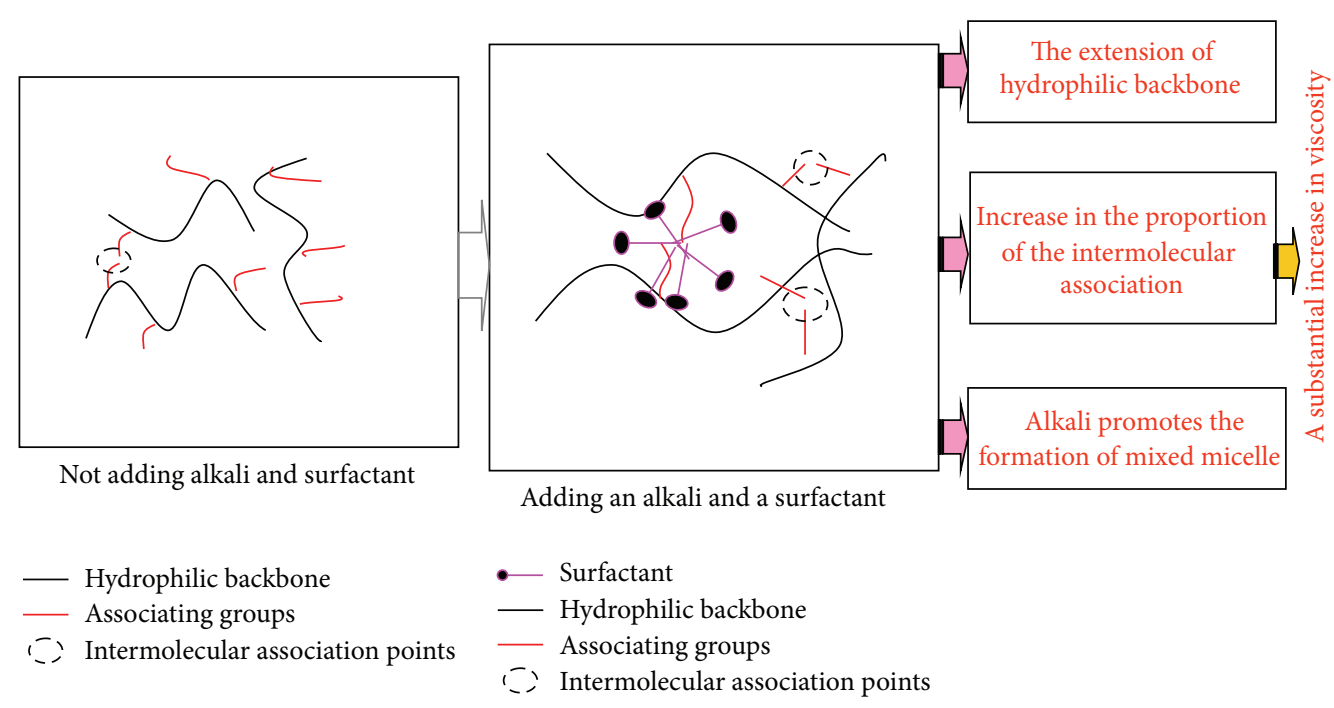

FIGURE 12: The effect of alkali mixed surfactant on the status of the associating polymer solution.

Because the base can promote the hydrolysis of the amide groups on the main chain of the associating polymer [20], it has less influence than $\mathrm{NaCl}$ on the viscosity of the associating polymer solution.

In the presence of both the base and the surfactant, the viscosity of the resulting ASP system is increased substantially. This is mainly because, under basic conditions, the carboxylic acid groups on the hydrophilic main chain have a higher hydration degree, the carboxylic acid groups are completely dissociated, and the molecular chain is more extended. Consequently, the surfactant interacts with the associating groups to form more intermolecular associating micelles, which substantially increases the solution viscosity (see Figure 12). This viscosity enhancement is closely related to the type and composition of the surfactant. Because the heavy alkylbenzene sulfonate surfactant used in this study has a very complicated composition, the mechanism of viscosity enhancement in the ASP system needs to be further studied.

\section{Conclusions}

The associating polymer HAPAM has good compatibility with the Daqing surfactant. The HAPAM ASP flooding system has good interfacial tension. After the addition of base and surfactant, the viscosity of the HAPAM ASP flooding system is increased significantly. Compared with using the HPAM polymer $(\mathrm{Mw}=2.5 \mathrm{MDa})$, using HAPAM gives much better viscosity and can reduce polymer use by more than $40 \%$. The core series injection experiment shows that the HAPAM ASP flooding system has good injectivity for the Daqing class II reservoirs $\left(100-300 \times 10^{-3} \mu \mathrm{m}^{2}\right)$. The pressure gradient is uniform between the measurement points. The oil recovery can be improved by more than $25 \%$ on top of water flooding. Under basic conditions, the carboxylic acid groups on the hydrophilic main chain have a higher hydration degree, the carboxylic acid groups are completely dissociated, and the molecular chain is more extended. This promotes the interaction between the surfactant and the associating groups to form more intermolecular associating micelles, which substantially increase the solution viscosity.

\section{Acknowledgments}

This work was carried out as a part of the National Science and Technology Major Project, China (2011ZX05010-004). The authors are grateful for the financial support. 


\section{References}

[1] Y. J. Guo, J. X. Liu, X. M. Zhang et al., "Solution property investigation of combination flooding systems consisting of gemininon-ionic mixed surfactant and hydrophobically associating polyacrylamide for enhanced oil recovery," Energy and Fuels, vol. 26, no. 4, pp. 2116-2123, 2012.

[2] M. F. Nazar, S. S. Shah, and M. A. Khosa, "Microemulsions in enhanced oil recovery: a review," Petroleum Science and Technology, vol. 29, no. 13, pp. 1353-1365, 2011.

[3] G. Shutang and G. Qiang, "Recent progress and evaluation of ASP flooding for EOR in Daqing oil field," in Proceedings of the SPE EOR Conference at Oil and Gas West Asia, pp. 48-54, Muscat, Oman, April 2010.

[4] H. Dijk, M. A. Buijse, J. Nieuwerf et al., "Salym Chemical EOR Project, integration leads the way to success," in Proceedings of the SPE Russian Oil and Gas Technical Conference and Exhibition, pp. 885-900, Moscow, Russia, October 2010.

[5] M. A. Buijse, R. M. Prelicz, J. R. Barnes, and C. Cosmo, "Application of internal olefin sulfonates and other surfactants to EOR. Part 2: the design and execution of an ASP field test," in Proceedings of the 17th SPE Improved Oil Recovery Symposium (IOR '10), pp. 706-717, Tulsa, Okla, USA, April 2010.

[6] J. Hou, Z. Liu, S. Zhang, X. Yue, and J. Yang, "The role of viscoelasticity of alkali/surfactant/polymer solutions in enhanced oil recovery," Journal of Petroleum Science and Engineering, vol. 47, no. 3-4, pp. 219-235, 2005.

[7] H. Zhang, M. Dong, and S. Zhao, "Which one is more important in chemical flooding for enhanced court heavy oil recovery, lowering interfacial tension or reducing water mobility?" Energy and Fuels, vol. 24, no. 3, pp. 1829-1836, 2010.

[8] W. M. Stoll, H. Al Shureqi, J. Finol et al., "Alkaline/surfactant/polymer flood: from the laboratory to the field," $S P E$ Reservoir Evaluation and Engineering, vol. 14, no. 6, pp. 702-712, 2011.

[9] C. S. Gregersen, M. Kazempour, and V. Alvarado, "ASP design for the Minnelusa formation under low-salinity conditions: impacts of anhydrite on ASP performance," Fuel, vol. 105, pp. 368-382, 2013.

[10] Q. Hou, G. Jian, M. A. Desheng, and Z. Wang, "Current development and application of chemical combination flooding technique," Petroleum Exploration and Development, vol. 40, no. 1, pp. 96-103, 2013.

[11] K. Panthi and K. K. Mohanty, "Effect of alkaline preflush in an alkaline-surfactant-polymer flood," Energy \& Fuels, vol. 27, no. 2, pp. 764-771, 2013.

[12] R. Kumar and K. K. Mohanty, "ASP flooding of viscous oils," in Proceedings of the SPE Annual Technical Conference and Exhibition (ATCE '10), pp. 4493-4503, Florence, Italy, September 2010.

[13] M. A. Bataweel and H. A. Nasr-El-Din, "Minimizing scale precipitation in carbonate cores caused by alkalis in ASP flooding in high salinity/high temperature applications," in Proceedings of the SPE International Symposium on Oilfield Chemistry, pp. 849-863, The Woodlands, Tex, USA, April 2011.

[14] K. Zhang and J. S. Qin, "The effect of alkali and surfactant on polymer molecular structure," Petroleum Science and Technology, vol. 29, no. 2, pp. 183-191, 2011.

[15] B. Wang, T. Wu, Y. Li et al., "The effects of oil displacement agents on the stability of water produced from ASP (alkaline/ surfactant/polymer) flooding," Colloids and Surfaces A, vol. 379, no. 1-3, pp. 121-126, 2011.
[16] L. Daoshan, L. Shouliang, L. Yi, and W. Demin, “The effect of biosurfactant on the interfacial tension and adsorption loss of surfactant in ASP flooding," Colloids and Surfaces A, vol. 244, no. 1-3, pp. 53-60, 2004.

[17] F. Chen, H. Jiang, X. Bai, and W. Zheng, "Evaluation the performance of sodium metaborate as a novel alkali in alkali/ surfactant/polymer flooding," Journal of Industrial and Engineering Chemistry, vol. 19, no. 2, pp. 450-457, 2013.

[18] W. Zhou, J. Zhang, M. Han et al., "Application of hydrophobically associating water-soluble polymer for polymer flooding in China offshore heavy oilfield," in Proceedings of the International Petroleum Technology Conference (IPTC '07), pp. 1449-1453, Dubai, United Arab Emirates, December 2007.

[19] Z. Huang, H. Lu, and Y. He, "Amphoteric hydrophobic associative polymer: I synthesis, solution properties and effect on solution properties of surfactant," Colloid and Polymer Science, vol. 285, no. 3, pp. 365-370, 2006.

[20] A. Samanta, A. Bera, K. Ojha, and A. Mandal, "Effects of alkali, salts, and surfactant on rheological behavior of partially hydrolyzed polyacrylamide solutions," Journal of Chemical and Engineering Data, vol. 55, no. 10, pp. 4315-4322, 2010. 

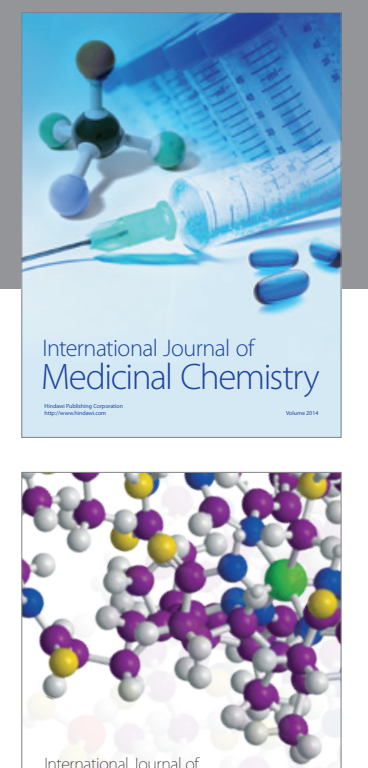

\section{Carbohydrate} Chemistry

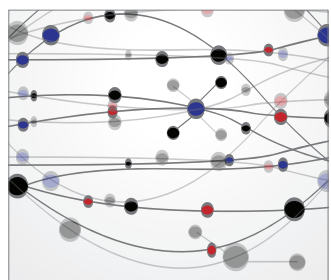

The Scientific World Journal
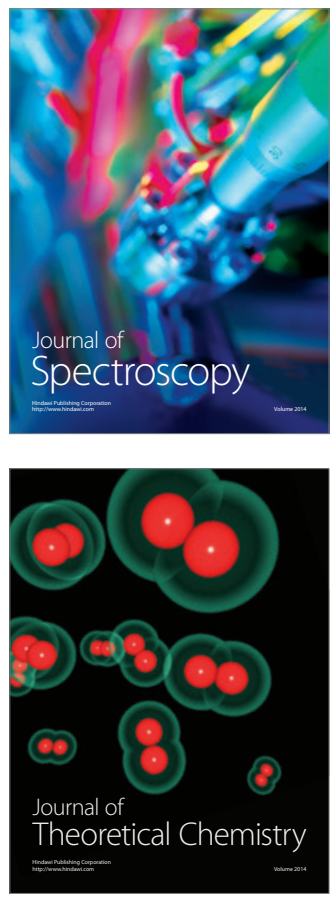
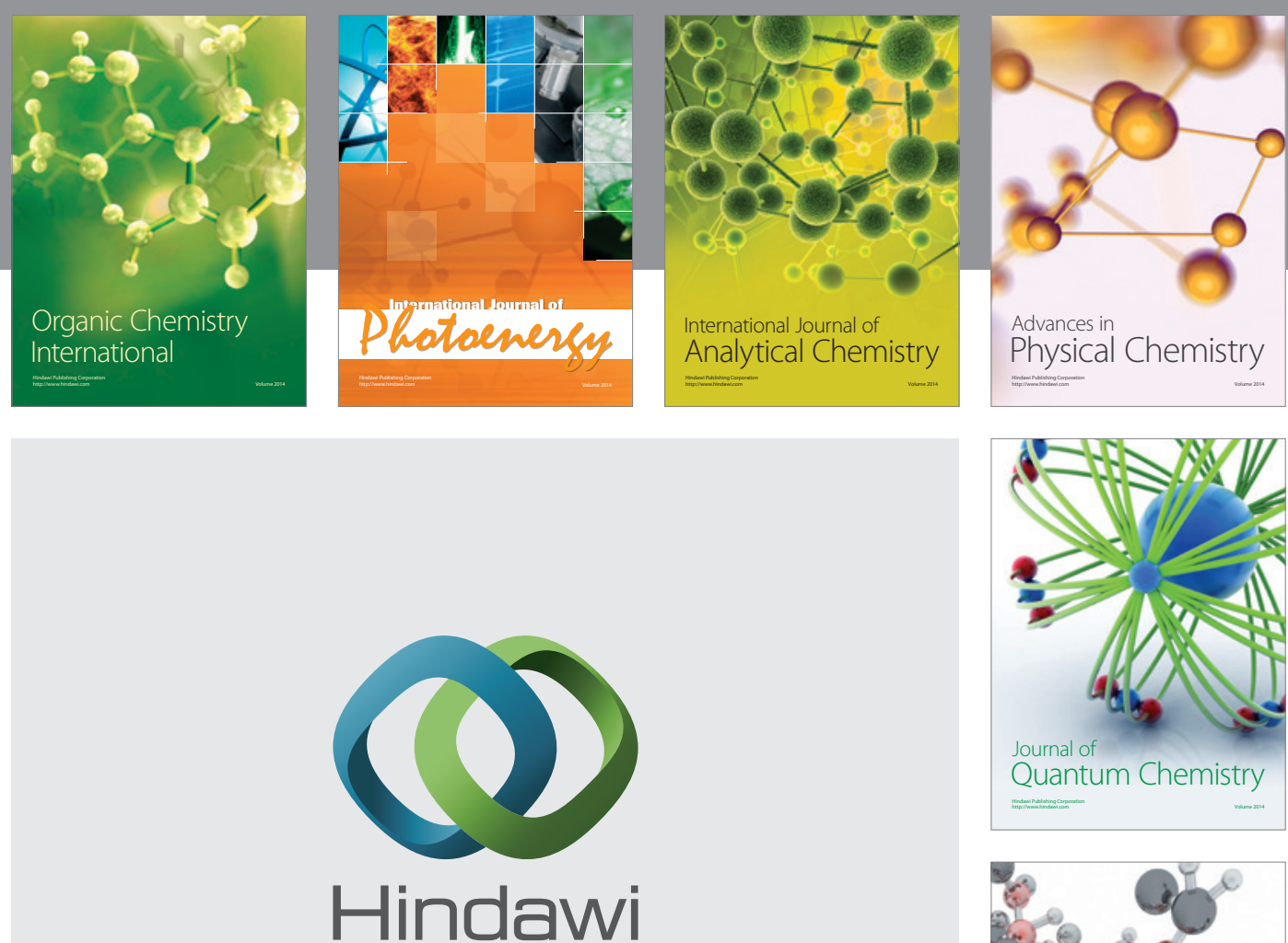

Submit your manuscripts at

http://www.hindawi.com

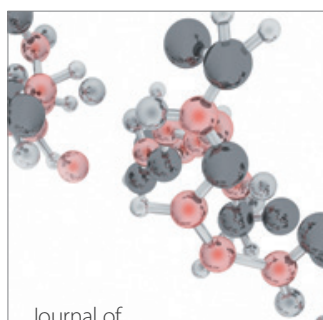

Analytical Methods

in Chemistry

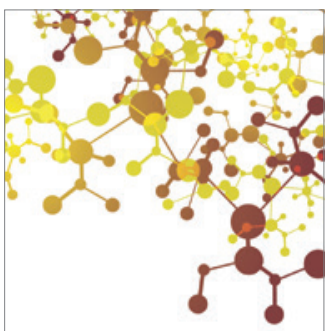

Journal of

Applied Chemistry

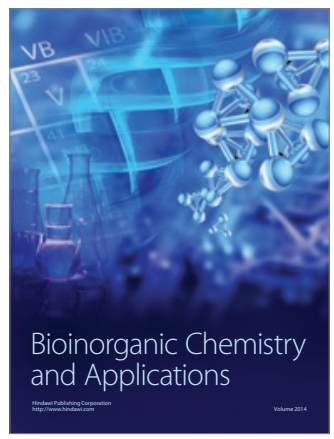

Inorganic Chemistry
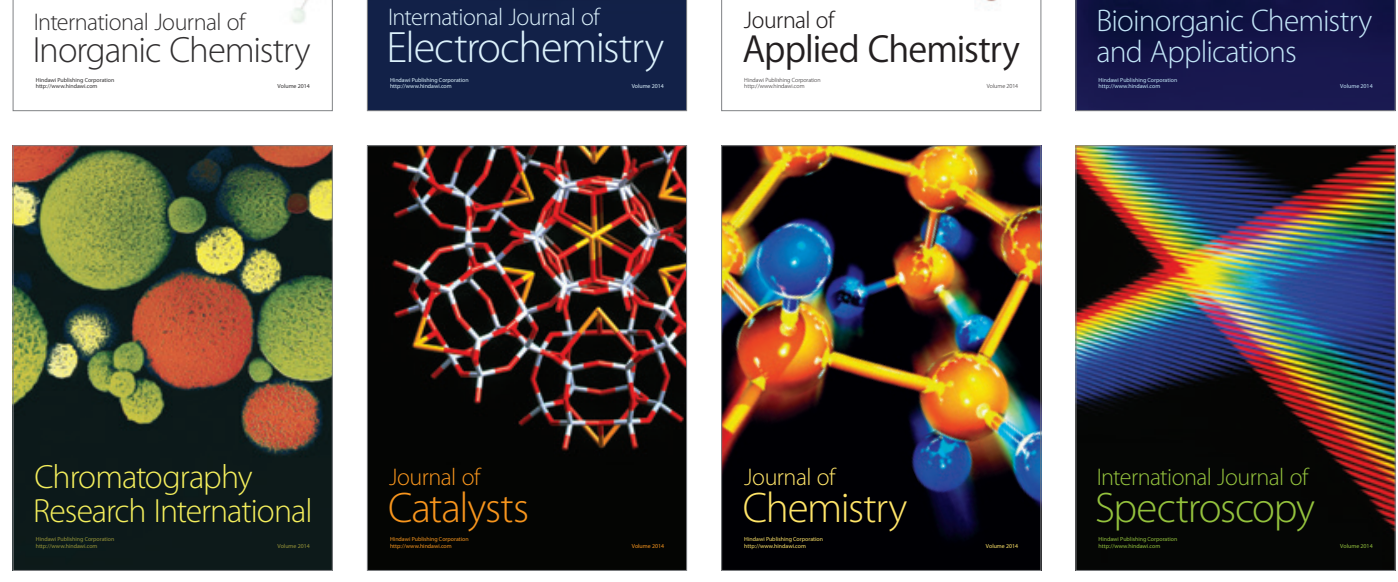